\title{
El uso concomitante de inhibidores de la bomba de protones y clopidogrel se asoció con recurrencia de infarto agudo de miocardio
}

Concomitant use of proton pump inhibitors and clopidogrel was associated with recurrent acute myocardial infarction

Juurlink D. y col. CMAJ 2009;180(7):713-8

\section{Objetivo}

Determinar la asociación entre el uso de Inhibidores de la Bomba de Protones (IBP) e Infarto Agudo de Miocardio (IAM) recurrente, en pacientes tratados con clopidogrel.

\section{Diseño y lugar}

Estudio de casos y controles* anidado* en una cohorte de Ontario, Canadá (2002 a 2007).

\section{Medición de resultados principales}

Cohorte de mayores de 65 años que contaran con el registro de uso y adherencia al tratamiento con clopidogrel -luego de haber sufrido un IAM- con comienzo del mismo dentro de los tres días del alta hospitalaria. El principal criterio de exclusión fue el uso de clopidogrel, ticlopidina o dipiridamol durante el año previo a IAM índice. Se definió como casos a aquellos pacientes fallecidos o re- internados por IAM recurrente dentro de los 90 días del primer ingreso (fecha índice). Los pacientes de la misma cohorte que no fueron re-admitidos formaron parte del grupo control. Los mismos fueron apareados* por edad, necesidad de reperfusión, fecha de alta y probabilidad estimada de mortalidad. En ambos grupos se investigó la exposición actual (dentro de los 30 días a la fecha índice) previa (31 a 90 días) y remota (más de 90 días) a IBP.

\section{Resultados Principales}

Se identificaron 13.636 pacientes (55,6\% varones). La prescripción concomitante de IBP fue del $31 \% ; 782$ pacientes reingresaron por IAM dentro de los 90 días del alta y de estos, el 93,9\% (734) fueron apareados con al menos un control. Se halló una asociación significativa entre la re-admisión debido a IAM y el uso concurrente de IBP, excepto para el pantoprazol. Ver tabla 1.

Tabla 1: asociación entre la exposición a IBP e infarto agudo de miocardio en pacientes tratados con clopidogrel.

\begin{tabular}{c|c|c|c}
\multirow{2}{*}{ Exposición a IBP } & \multicolumn{2}{|c|}{ Número de pacientes y (\%) } & \multicolumn{2}{c}{$\begin{array}{c}\text { OR ajustado* } \\
\text { (IC95\%) }\end{array}$} \\
\cline { 2 - 4 } Ninguna & Casos $\mathbf{n = 7 3 4}$ & Controles $\mathbf{n}=\mathbf{2 0 5 7}$ & 1,00 \\
\hline Hace menos de 30 días & $448(61,0)$ & $1317(64,0)$ & $1,27(1,03$ a 1,57$)$ \\
\hline Pantoprazol & $194(26,4)$ & $424(20,6)$ & $1,02(0,70$ a 1,47$)$ \\
\hline Otros IBP & $46(6,3)$ & $125(6,1)$ & $1,40(1,10$ a 1,77$)$ \\
\hline Previa (31-90 días) & $148(20,2)$ & $299(14,5)$ & $0,86(0,63$ a 1,19$)$ \\
\hline Remota (91-180 días) & $63(8,6)$ & $195(9,5)$ & $0,81(0,46$ a 1,41$)$ \\
\hline
\end{tabular}

* Ajustado por edad, sexo, nivel de ingreso estimado, índice de comorbilidad de Charlson, estadía hospitalaria, comorbilidades y medicación crónica. y Omeprazol, rabeprazol, lanzoprazol.

\section{Conclusiones}

El uso concomitante de IBP y clopidogrel se asoció con un incremento del riesgo de recurrencia de IAM dentro de los primeros tres meses de un episodio previo. Este efecto no se observó con el uso de pantoprazol.
Palabras claves: Etiología, inhibidores de la bomba de protones, clopidogrel, infarto agudo de miocardio recurrente.

Key words: etiology, proton pump inhibitors, clopidogrel, recurrent acute myocardial infarction.

Fuente de financiamiento: Ministerio de Salud y Cuidado Longitudinal de Ontario, Instituto Canadiense de Investigación en Salud (entre otras fuentes institucionales).

\section{Comentario}

El clopidogrel es una pro-droga y actúa gracias a la conversión hepática en su metabolito activo, por acción de la isoenzima citocromo P450 2C19. Recientemente la Agencia Europea de Medicamentos (sigla en inglés, EMEA) y la Administración Norteamericana de Drogas y Alimentos (sigla en Inglés, FDA) han advertido de su posible interacción con IBP, sugiriendo la necesidad de reevaluar el inicio o continuidad de los IBP en pacientes tratados con clopidogrel ${ }^{1}$, o de desaconsejar su uso conjunto $^{2}$, sin requerir el retiro del mercado de alguna de las drogas implicadas. En Argentina la Administración Nacional de Medicamentos, Alimentos y Tecnología Medica (ANMAT) aún no se ha expedido al respecto. Los resultados publicados en este estudio ofrecen evidencia en contra del uso conjunto de ambos medicamentos en pacientes con un IAM reciente, que sin embargo debe manejarse con cautela ${ }^{3}$. En ese sentido, vale destacar que por su diseño observacional, las poblaciones comparadas no fueron homogéneas ya que el grupo de casos Juan Pablo Corso [ Médico Especialista en Cardiología, Hospital Andrés Isola. Puerto Madryn, Chubut.juanpablo.corso@gmail.com ]

Recibido el 10/05/2009 y aceptado el 14/07/2009 Corso JP. El uso concomitante de inhibidores de la bomba de protones y clopidogrel se asoció con el infarto agudo de miocardio recurrente. Evid. actual. práct. ambul; 12(4):132, Oct-Dic.2009. Comentado de: Juurlink y col. A population-based study of the drug interaction between proton pump inhibitors and clopidogrel. CMAJ 2009;180(7):713-8. PMID: 19176635. Disponible en URL: http://www.cmaj.ca/cgi/reprint/180/7/713

\section{Referencias}

1 US Food and Drug Administration. Early Communication about an Ongoing Safety Review of clopidogrel bisulfate (marketed as Plavix) [en línea]. Actualizado al 13/0572009. Disponible en URL:

http://www.fda.gov/Drugs/DrugSafety/PostmarketDrugSafetylnformationforPatientsandProviders/DrugSafetylnformationforHeathcareProfessionals/ucm079520.htm (último acceso 14/07/2009).

2. European Medicines Agency. Public statement on possible interaction between clopidogrel and proton-pump inhibitors (en línea). Londres, Mayo 2009. Disponible en URL: http://www.emea.europa.eu/humandocs/PDFs/EPAR/Plavix/32895609en.pdf (último acceso 14/07/2009).

3. The Heart.org (Sitio web). Hughes S. EMEA issues warning on possible clopidogrel-PPI interaction, but is there really a problem?. Junio 2009. Disponible en URL: http://www.theheart.org/article/980779.do (último acceso 14/07/2009). 